\title{
Quantum induced broadening: A challenge for cosmic neutrino background discovery
}

\author{
Shmuel Nussinov ${ }^{1}$ and Zohar Nussinov ${ }^{2}$ \\ ${ }^{1}$ School of Physics and Astronomy, Tel Aviv University, 6997801 Tel Aviv, Israel \\ ${ }^{2}$ Department of Physics, Washington University, St. Louis, Missouri 63130, USA
}

(Received 15 August 2021; accepted 12 January 2022; published 1 February 2022)

\begin{abstract}
A recent preprint by Cheipesh et al. pointed out that the zero-point motion of tritium atoms bound to graphene may blur the measured energies of $\beta$ electrons. Smearing due to zero point motion is well known. Such an effect features in studies of the $\beta$ spectrum expected in experiments like KATRIN using diatomic tritium. The recent preprint may, however, challenge new planned experiments seeking to discover the cosmic neutrino background (CNB) neutrinos (and/or other neutrinos of masses smaller than $0.1 \mathrm{eV}$ ), which plan to use tritium adsorbed onto graphene or other materials. Our paper clarifies these issues and examines the more general problem of smearing induced by quantum uncertainty. We find that the effect of Cheipesh et al. is reduced considerably. The importance of the chemical evolution of the ${ }^{3} \mathrm{H}$ atom hosting the tritium nucleus into a tightly bound neutral ${ }^{3} \mathrm{He}$ atom is emphasized. We estimate the excess blurring caused by the dense spectrum near the lowest state of the graphene or other hosts of the tritium atom, generated by the electronic response to the "sudden" escape of the $\beta$ electron. Our analysis suggests yet larger effects and difficulties facing many experiments searching for small mass neutrinos. We speculate on a possible experimental setup, which could minimize quantum broadening.
\end{abstract}

DOI: 10.1103/PhysRevD.105.043502

\section{INTRODUCTION}

Smearing due to zero point motion is well known and features in studies of the $\beta$ spectrum expected in experiments like KATRIN using tritium bound in diatomic $\left(T_{2}\right)$ molecules [1,2]. A recent paper by Yevhenia et al. [3] pointed out that such effects may undermine Ptolomey type [4] experiments that plan to use tritium adsorbed on graphene sheets. These experiments [4] are designed to discover the cosmological neutrino background (CNB) by careful studies of the near and beyond the end point spectrum of electrons emerging from the $\beta$ decay of tritium atoms and from the inverse $\beta$ process where a CNB neutrino is captured by the tritium.

An analysis of the CMB data of the Planck Collaboration along with other cosmological data suggested a most stringent bound on the sum of the masses of the three neutrinos [5],

$$
\sum_{i} m\left(\nu_{i}\right) \leq 0.12 \mathrm{eV}
$$

Published by the American Physical Society under the terms of the Creative Commons Attribution 4.0 International license. Further distribution of this work must maintain attribution to the author(s) and the published article's title, journal citation, and DOI. Funded by SCOAP ${ }^{3}$. suggesting that the mass of the lightest neutrino of interest is less than $40 \mathrm{meV}$.

The lofty goal of CNB discovery faces immense challenges. Detailed calculations [6] indicate that, even in largescale experiments, only a handful of the $\beta$ electrons would land in the region of interest beyond the end point of the continuous spectrum. These electrons are displaced relative to the $Q \simeq 18.6 \mathrm{KeV}$ end point of the continuum spectrum of the decay electrons by twice the mass of the background neutrino. If this shift is by less than $0.1 \mathrm{eV}$ then a very high energy resolution will be needed in order to separate the electrons from the CNB capture from those in the much higher, steeply falling, continuous tritium $\beta$ decay spectrum.

The captivating concept underlying these experiments is that in order to discover the CNB, we do not require the precise values of the expected and/or measured energies at and above the end point of the tritium $\beta$ decay. Rather, all that is needed is that the measured distribution $F(\epsilon(\beta(e)))$ of the energy of the $\beta$ electrons will display the pattern shown in Ref. [3], reproduced in Fig. 1, with approximately a dozen electrons in a peak (or two peaks) above the observed endpoint. This holds for any effect shifting the energy $\epsilon(e)$ in the end point region so long as the shift has a fixed, sharp, value. This also applies for ongoing experiments like KATRIN searching for small distortions of the continuum spectrum near the endpoint due to small neutrino masses. In assessing the prospects of such experiments for detecting $\mathrm{CNB}$, we need then to worry only about 


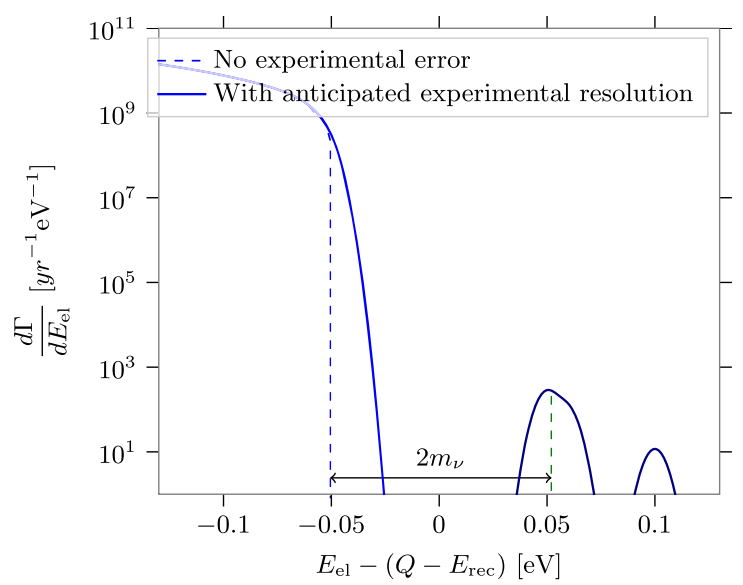

FIG. 1. Reproduced from [3]. The energy spectrum of tritium $\beta$ decay in the end point region and the peaks due to CNB captures beyond it. Here, the two CNB masses are $m(2)=2 m(1)=$ $0.1 \mathrm{eV}$. In the figure, for emphasis, the separation of the peaks exceeds $\delta\left(m^{2}\right) /(m(2)+m(1))$ (whereas it should be identically equal to the latter ratio). The square difference $\delta\left(m^{2}\right) \equiv m^{2}(2)-$ $m^{2}(1)$ was measured to be $\sim 8.6 \times 10^{-5} \mathrm{eV}^{2}$. For illustrative purposes, the area under the first peak, proportional to $\left|U_{e 1}\right|^{2}$ (the mixing of $\nu_{e}$ and $\nu_{1}$ ), was exaggerated relative to the area under the second peak (proportional to $\left|U_{e 2}\right|^{2}$ ).

variations leading to the blurring of the energy distribution of the $\beta$ electrons around the above noted $Q \simeq 18.6 \mathrm{KeV}$ end point.

Various "technical" sources of broadening can be handled. The binding energy $(B)$ of the adsorbed ${ }^{3} \mathrm{H}$ atom of $\sim 1.85 \mathrm{eV}$ exceeds the resolution needed to establish the CNB induced peak(s) and variations of the bindings of different tritium atoms would be harmful. However, this problem may be avoided. For instance, in pristine graphene patches, all binding sites are identical with each ${ }^{3} \mathrm{H}$ atom being equally bound [7]. In this situation, the uniformity disappears at times (as measured from the start of the experiments) approaching the half life of tritium ( $\tau \sim 13$ years). At such times, we may find different local arrangements of surviving tritium atoms. The random set of tritium atoms, which has decayed, is transmuted into helium ions or helium atoms that often escape the graphene sheet. The fact that neighboring tritium atoms may spontaneously tunnel and rearrange into a diatomic $T_{2}$ molecule and evaporate limits on the allowed density of packing. Thermal doppler broadening in this and gaseous setups can be minimized by cooling. Finally, the standard energy losses by ionization of the $\beta$ electrons as they traverse the $\mathrm{O}$ (angstrom) graphene sheet are negligible, and on average, only one in $\sim 10^{6} \beta$ electrons will interact on its "way out" and lose energy by ionization.

In the upcoming discussion, we will employ upper (and lower) case letters to denote quantities related to the heavy ${ }^{3} \mathrm{H},{ }^{3} \mathrm{He}^{+}$, and ${ }^{3} \mathrm{He}$ atoms (and with the light $\beta$ electron and $\nu)$, respectively. We will often implicitly set $c$ to unity.
However, in order to highlight zero point quantum effects, which form a focus of our work, we will keep factors of $\hbar$.

The authors of [3] recall the theory underscoring irreducible "noise" due to zero point motion. Their argument is presented in an idealized context where the hosting graphene merely provides a static binding potential which, near its minimum, is approximated by a harmonic oscillator potential. The Heisenberg uncertainty principle implies, in the harmonic approximation near the minimum, a momentum spread $\hbar \vec{K}$ of the tritium atom that is set by the reciprocal of its localization length,

$$
\Delta K_{i} \sim \frac{1}{2 \Delta R_{i}}
$$

Here, $i$ labels Cartesian components of the momentum and position vectors. In the (Gaussian) harmonic oscillator ground state, the more general Heisenberg uncertainty relation inequality is saturated with the above factor of $1 / 2$ in Eq. (2). In this instance, the approximate equality in Eq. (2) is precise and estimates made using this relation become exact. The chemisorption potential responsible for the deeper binding of the tritium to the graphene is most constraining in the $Z$ direction (with the $Z$ direction defined to be perpendicular to the $X Y$ plane of the graphene sheet). The average (as computed by $a b$ initio calculations of various groups [8-10]) of the rigidity $\kappa$ of the effective harmonic potential near the minimum of the actual potential then yields an estimate of the amplitude of the vertical oscillations of $\Delta Z \sim 0.13 \mathrm{~A}$. Due to the relatively large ${ }^{3} \mathrm{H}$ mass, this spatial fluctuation scale is much smaller than the length of the $\mathrm{CH}$ bond - the true distance between the ${ }^{3} \mathrm{H}$ and the carbon atom just below (or above) it, of $\sim 1.2 \mathrm{~A}$. The harmonic approximation holds over this $0.13 \mathrm{~A}$ interval. Employing Eq. (2), this localization yields $\hbar c \Delta K_{Z} \sim 7.7 \mathrm{KeV}$. With this $K_{Z}$, the final ${ }^{3} \mathrm{He}^{+}$ion and electron are emitted from a source moving with a spread of velocities,

$$
\Delta V_{Z}=\frac{\hbar \Delta K_{Z}}{M\left({ }^{3} \mathrm{H}\right)} \sim 2.5 \times 10^{-6} c .
$$

A shallow "migration" potential limits the lateral motion along the $\mathrm{X}$ and $\mathrm{Y}$ directions. The coefficients of the restoring forces $\kappa_{X}=\kappa_{Y}$ are an order of magnitude smaller than $\kappa_{Z}$. The resulting standard deviations of the velocity components of the tritium $\Delta V_{X}=\Delta V_{Y} \sim \kappa^{1 / 4} \Delta V_{Z} \sim 10^{-1 / 4} \Delta V_{Z}$. The varying boosts then smear the energy distribution $F(\epsilon(\beta(e)))$ of the emitted $\beta$ electron by

$$
\begin{aligned}
\delta \epsilon(\beta(e)) & =\Delta \vec{V} \cdot \vec{p}(e) \\
& =\Delta V_{X} p_{x}(e)+\Delta V_{Y} p_{y}(e)+\Delta V_{Z} p_{x}(z) \\
& =p(e)\left(n_{x} \Delta V_{X}+n_{y} \Delta V_{Y}+n_{z} \Delta V_{Z}\right),
\end{aligned}
$$


where $n_{x}, n_{y}$, and $n_{z}$ are the direction cosines of the electron momentum. The magnitude of the momentum of the electron $|\vec{p}(e)|$ at (and near) the end point is

$$
p(e)=[2 m(e) Q]^{1 / 2} \sim 137 \mathrm{KeV},
$$

where we inserted $Q \sim 18.6 \mathrm{KeV}$ for the end point of the $\beta$ electron energy distribution. In the following, we will denote the angular averages by $\langle-\rangle$. Squaring Eq. (4) and omitting the mixed Cartesian component terms (since those average to zero),

$$
\begin{aligned}
\sqrt{\left\langle(\delta \epsilon(\beta(e)))^{2}\right\rangle} & =p(e) \Delta V_{Z} \sqrt{\left(n_{x}^{2}+n_{y}^{2}\right)\left(10^{-1 / 4}\right)^{2}+n_{z}^{2}} \\
& \sim 0.544 p(e) \Delta V_{Z}=0.186 \mathrm{eV} .
\end{aligned}
$$

In Eq. (6), we used $n_{x}^{2}+n_{y}^{2}+n_{z}^{2}=1$ and $\left\langle n_{z}^{2}\right\rangle=1 / 3$ and, in the last equality, we substituted the standard deviation of the tritium $Z$ velocity component of Eq. (3). This final result for the ZPM induced blurring is significantly lower than that suggested by [3]. The reasons for this discrepancy are twofold: (a) a factor of $1 / 2$ due to the saturation of the Heisenberg inequality omitted in [3] and (b) a factor of $1 / \sqrt{3}$ from angular averaging over the uniformly distributed directions of $\vec{p}(e)$. Nonetheless, the reduced smearing of Eq. (6) still exceeds the separation between the end point of the spectrum and the putative tiny peak(s) due to the CNB neutrino absorption if the mass of the latter is smaller than $0.1 \mathrm{eV}$.

Blurring effects of the $\beta$ electron are associated with a dense spectrum of energies of the system hosting the initial tritium nucleus near its lowest energy state induced by the escape of the $\beta$ electron. Only final states where the initial ${ }^{3} \mathrm{H}$ atom converted into the tightly bound neutral ${ }^{3} \mathrm{He}$ atom are associated with the sought, most energetic, $\beta$ electrons.

In the current work, we will discuss, in some detail, three possible settings. Specifically, we will examine what transpires when the host system is (a) the initial ${ }^{3} \mathrm{H}$ atom, (b) an ${ }^{3} \mathrm{H}-{ }^{3} \mathrm{H}$ molecule for a tritium source that is molecular or (c) the far more complex situation in which the ${ }^{3} \mathrm{H}$ atom is adsorbed on a graphene sheet. Our general framework of a "dense host energy spectrum" includes the smearing due to the ZPM, where the relevant degree of freedom is the linear motion of the ${ }^{3} \mathrm{He}^{+}$ion or the ${ }^{3} \mathrm{H}$ atom generated in the $\beta$ process. With only a single degree of freedom involved, this can be treated exactly.

Rather than merely viewing the graphene as the source of the static potential binding the tritium, we explore the dynamics of the graphene. This involves both phonon (i.e., normal mode vibrations of the carbon ions in the twodimensional graphene lattice) and electronic excitations. Blurring arising from phonon type oscillations is discussed in Sec. III as it ties with smearing triggered by the tritium ZPM that is elaborated in Sec. II. We find a small excess smearing (by $\sim 12 \%$ ) due to this effect. By contrast, the estimates of broadening due to electronic excitations suggest large effects. Thus, these excitations constitute a formidable barrier for experiments using tritium attached to graphene or other surfaces to discover the CNB neutrinos. Finally, we briefly suggest a highly speculative scheme of attaching tritium atoms to a surface which may reduce broadening effects due to quantum uncertainties.

\section{KINEMATICS OF THE CNB CAPTURE AND SMEARING BY BOOSTS DUE TO ZERO POINT FLUCTUATIONS}

The CNB neutrino in the capture reaction,

$$
\nu(e)+{ }^{3} \mathrm{H} \rightarrow e^{-}+{ }^{3} \mathrm{He}^{+},
$$

and the antineutrino emitted in the $\beta$ decay process,

$$
{ }^{3} \mathrm{H} \rightarrow e^{-}+{ }^{3} \mathrm{He}^{+}+\bar{\nu}(e),
$$

are eigenstates of electron flavor $\nu(e)$. In the "normal hierarchy" of neutrino masses, $\nu(e)$ mixes mainly with the lower mass eigenstates $\nu(1)$ and $\nu(2)$, which have a small, measured, $m^{2}$ splitting [11],

$$
\delta\left(m^{2}\right)_{1,2}=[m(2)]^{2}-[m(1)]^{2} \sim 8.6 \times 10^{-5}(\mathrm{eV})^{2} .
$$

Detecting two peaks beyond the end point, at locations and relative strengths fixed by $\delta\left(\mathrm{m}^{2}\right)$ and by the measured mixings, respectively, may be invaluable for confirming a discovery of the CNB. The mass $m(1)$ of the lightest neutrino is unknown. Assuming $m(1) \sim m(2)-m(1)$ and that $m(2) \sim 2 m(1) \sim 50 \mathrm{meV}$ leads to a $\beta$ spectrum from tritium decays and CNB neutrino capture reactions as given in Fig. 1 in Ref. [3] (reproduced in Fig. 1). For higher $m(1)$ values, the need to keep $\delta\left(m^{2}\right)$ fixed, forces $[m(2)-m(1)]$ to decrease and the two peaks merge at $\sim 2 m(1)$ above the end point. In the following, we therefore simplify the analysis by considering only one effective light neutrino $\nu_{1}$ (instead of two) with a large mixing $\left|U_{e 1}\right|^{2}+\left|U_{e 2}\right|^{2} \sim 0.95$ (close to the maximal value of one (for the full unitary neutrino mixing matrix $U$ )) with only $\nu_{1}$ and its associated single peak (instead of two peaks) above the end point.

The kinematics of the $2 \rightarrow 2$ body CNB capture process is greatly simplified by having the ${ }^{3} \mathrm{H}$ essentially at rest and the incoming nonrelativistic CNB neutrino carry almost zero momentum. The neutrino then just adds its rest mass $m(1)$ to that of the tritium to form an intermediate state I at rest in the laboratory frame of mass,

$$
M(I)=M\left({ }^{3} \mathrm{H}\right)+m(1) .
$$

The weak interactions transform this state into the final electron and the ${ }^{3} \mathrm{He}^{+}$ion whose center of mass frame is also at rest relative to the laboratory frame [12]. The outgoing $\beta$ electron and ${ }^{3} \mathrm{He}^{+}$ion are then ejected in opposite directions with momenta of equal magnitude, 


$$
p(\beta(e))=p=P\left({ }^{3} \mathrm{He}^{+}\right)=P .
$$

Energy conservation implies that

$$
\begin{aligned}
\epsilon(e)+E\left({ }^{3} \mathrm{He}^{+}\right)= & {\left[M\left({ }^{3} \mathrm{H}\right)+m(1)+E\left({ }^{3} \mathrm{H}\right)\right] } \\
& -M\left({ }^{3} \mathrm{He}^{+}\right)-m(e)+\delta E_{\text {atomic }},
\end{aligned}
$$

where $E\left({ }^{3} \mathrm{H}\right), E\left({ }^{3} \mathrm{He}^{+}\right)$, and $\epsilon(e)$ refer to kinetic energies and $\delta E_{\text {atomic }}$ is the change of bindings of the atomic electron due to the transition from the initial $Z=1$ hydrogen to the final $Z=2$ hydrogen-like helium ion. The on shell conditions for the final electron and helium ion,

$$
\begin{aligned}
p(e) & =\left((m(e)+\epsilon(e))^{2}-m(e)^{2}\right)^{1 / 2}, \\
P\left({ }^{3} \mathrm{H}^{+}\right) & =\left[\left(M^{3} \mathrm{He}^{+}+E_{{ }^{3} \mathrm{He}^{+}}\right)^{2}-\left(M^{3} \mathrm{He}^{+}\right)^{2}\right]^{1 / 2},
\end{aligned}
$$

then prescribe sharp values of the kinetic energies of the outgoing electron and ${ }^{3} \mathrm{He}^{+}$helium ion. Namely,

$$
\begin{aligned}
& \epsilon(e)+m(e) \\
& =\frac{\left[M\left({ }^{3} \mathrm{H}\right)+m(1)\right]^{2}+\left[M\left({ }^{3} \mathrm{He}^{+}\right)\right]^{2}-[m(e)]^{2}}{2\left(M\left({ }^{3} \mathrm{H}\right)+m(1)\right)} \\
& \quad+\delta E_{\text {atomic }},
\end{aligned}
$$

and

$$
\begin{aligned}
& E\left({ }^{3} \mathrm{He}^{+}\right)+M\left({ }^{3} \mathrm{He}^{+}\right) \\
& \quad=\frac{\left[M\left({ }^{3} \mathrm{H}\right)+m(1)\right]^{2}-\left[M\left({ }^{3} \mathrm{He}^{+}\right)\right]^{2}-[m(e)]^{2}}{2\left(M\left({ }^{3} \mathrm{H}\right)+m(1)\right)} .
\end{aligned}
$$

The above equations express, in terms of the initial kinetic energies, the familiar relativistic kinematics of the decay of a particle of mass $M$ into particles of masses $M(1), m(2)$ with total energies $E(M(1))=\frac{M^{2}+M^{2}(1)-m^{2}(2)}{2 M}$ and $\epsilon(m(2))=\frac{M^{2}-M^{2}(1)+m^{2}(2)}{2 M}$.

If the tritium ${ }^{3} \mathrm{H}$ atom interacting with the $\mathrm{CNB}$ neutrino is bound to, say, a graphene sheet, then the energy conservation relation will be modified to

$$
\begin{aligned}
\epsilon(e)+E\left[{ }^{3} \mathrm{H}^{+}\right]= & {\left[M\left({ }^{3} \mathrm{H}\right)-B+m(1)+\epsilon(\nu(1))\right] } \\
& -M\left({ }^{3} \mathrm{He}^{+}\right)-m(e)+\delta E_{\text {atomic }},
\end{aligned}
$$

where $B$ is the binding of ${ }^{3} \mathrm{H}$ to the graphene. This binding is smaller than the recoil kinetic energy of $\sim 3.5 \mathrm{eV}$ of the final ${ }^{3} \mathrm{He}^{+}$in the free decay, and we first discuss the case where the ${ }^{3} \mathrm{He}^{+}$escapes the graphene. We will first also follow [3], where all the effects of the host graphene were subsumed by a static potential $\mathcal{V}\left(\right.$ graphene $\left.{ }^{3} \mathrm{H}\right)=\mathcal{V}$.

Unlike the CNB neutrino capture on free ${ }^{3} \mathrm{H}$, the capture here is on a tritium atom, which is bound in a potential and can exchange momentum $\hbar \vec{K}$ with it. The system of
$I=C N B(\nu)+{ }^{3} \mathrm{H}$ and as well as the center mass frame of the final $\beta$ electron and helium ion are then boosted relative to the lab frame, where the energy $\epsilon(\beta(e))$ of the $\beta$ electron is measured. The resulting change of the energy of the outgoing $\beta$ electron depends on the magnitude and direction of $\vec{K}$ relative to the momentum of the electron. Thus, a broadening of the distribution $F(\epsilon(\beta(e)))$ of the final $\beta$ electron energies is expected from pure kinematic arguments.

In order to further assess the magnitude of the effect, dynamical information is required. Before the $\beta$ process happens, say at $t=0$, the ${ }^{3} \mathrm{H}$ atom is in the lowest bound state in the potential $\mathcal{V}$. In momentum space, we will represent the ground state of the ${ }^{3} \mathrm{H}$ atom by a wave function $\tilde{\psi}_{0}(\vec{K})$ - the Fourier transform of the ground wave function in configuration space. The associated momentum probability distribution is given by $F(\vec{K})=\left|\tilde{\psi}_{0}(\vec{K})\right|^{2}$. With the ${ }^{3} \mathrm{H}$ atom moving in its bound state with momenta $\vec{K}$ distributed according to $F(\vec{K})$ and corresponding velocities $\vec{V}=\hbar \vec{K} / M_{{ }^{3} \mathrm{H}}$, the boosted initial system I of the tritium ${ }^{3} \mathrm{H}$ with the CNB $\nu(1)$ resting on it, will decay into the final electron and ${ }^{3} \mathrm{H}^{+}$with each particle having the claimed spread of energies of $\pm \hbar \vec{p} \cdot \vec{K} / M$. Viewing the decaying tritium as a freely evolving wave packet, which seems to be implied above, would entail energy nonconservation. Each plane wave component of $\tilde{\psi}_{0}$ carries a kinetic energy $\hbar^{2} K^{2} /\left(2 M_{{ }^{3} \mathrm{H}}\right)$ adding up to the positive,

$$
\int d^{3} K F(\vec{K}) \frac{\hbar^{2} \vec{K}^{2}}{2 M_{3^{3}}} .
$$

Thus, both the final $e^{-}$and the ${ }^{3} \mathrm{He}^{+}$ion will have not only opposite energy shifts $\pm \vec{V} \cdot \vec{p}(e)$ relative to the situation of free atomic decay. Rather, they will appear to jointly have an extra kinetic energy not accounted for by the mass differences of the initial and final particles. This, however, is not the case. The tritium atoms with higher kinetic energies are, in a classical picture, located nearer to the minimum of, and deeper in, the binding potential. This attractive potential energy is to be subtracted from that of the outgoing ${ }^{3} \mathrm{He}^{+}$as it leaves the graphene sheet. If the potentials exerted by the graphene on the initial ${ }^{3} \mathrm{H}$ atom and the final ${ }^{3} \mathrm{He}^{+}$ion are the same,

$$
\mathcal{V}\left({ }^{3} \mathrm{H}-\text { graphene }\right)=\mathcal{V}^{\prime}\left({ }^{3} \mathrm{He}^{+}-\text {graphene }\right),
$$

then the net effect, also in the quantum case, amounts just to the binding energy $(-B)$ term in the energy conservation relation above.

It is instructive to rederive the smearing due to ZPM in a slightly different way. The oscillation frequencies $\omega_{i}=$ $\left(\kappa_{i} / M\right)^{1 / 2}$ and the zero mode energies are $\hbar \omega_{i} / 2$. In the harmonic oscillator eigenstates, the average kinetic energy is half of the total energy. Since the expectation of velocity components in stationary states vanish, the variance, 


$$
\left(\Delta V_{i}\right)^{2} \equiv\left\langle V_{i}^{2}\right\rangle-\left\langle V_{i}\right\rangle^{2}=\left\langle V_{i}^{2}\right\rangle=\frac{\hbar\left(\kappa_{i}\right)^{1 / 2}}{2 M^{3 / 2}}
$$

The contribution of the zero point oscillations in the $\mathrm{Z}$ direction perpendicular to the graphene sheet and those of the $\mathrm{X}$ and $\mathrm{Y}$ independent oscillations in the in plane simply add up, reflecting the separability of the harmonic oscillator problem in Cartesian coordinates. With the average of the $\kappa_{3}$ values for the chemisorption minimum computed by the three different groups of [8-10] being $\kappa_{3} \sim 3.5 \mathrm{eV} / \mathrm{A}^{2}$, we find that $\hbar \omega_{Z} \sim 0.07 \mathrm{eV}$. This recovers the earlier result of $\Delta V_{Z} \sim 2.5 \times 10^{-6} \mathrm{c}$ and the earlier derivation of the ZPM broadening follows again.

\section{PHONON EXCITATIONS}

We next proceed to discuss the effect of zero point oscillations of the carbon atoms in the graphene lattice. While the resulting effect is rather small, its derivation is, nonetheless, quite instructive. To estimate the effect, we use the Debye temperature. Graphene features notably different sound velocities [corresponding to vibrations in the graphene (XY) plane and in the $\mathrm{Z}$ direction] [13]. These phonon spectra are associated with two Debye temperatures, $T_{\text {Debye }}^{(1)}=2312 \mathrm{~K}$ and $T_{\text {Debye }}^{(2)}=1287 \mathrm{~K}$. The lower energy phonons (corresponding to the Debye temperature of $T_{\text {Debye }}^{(2)}$ ), associated with oscillations in the $Z$ direction, will form the focus of attention next. In a circular (or " $2 D$ spherical") Brillouin zone approximation (similar to that typically employed in the Debye model) for the 2D graphene sheet, the average phonon frequency (over all modes $\vec{k}$ ) associated with the $\mathrm{Z}$ direction,

$$
\hbar \omega_{\text {Average }} \sim \frac{2}{3} k_{B} T_{\text {Debye }}^{(2)} \sim 0.074 \mathrm{eV} .
$$

The two other phonon modes jointly carry almost 4 times as much energy as the $\mathrm{Z}$ mode. We note, however, that the ${ }^{3} \mathrm{H}$ atom is at $Z \sim \pm 1.2 \mathrm{~A}$ relative to the graphene sheet. Thus, small amplitude $\mathrm{X}$ and $\mathrm{Y}$ oscillations of the carbon atom that is connected to the tritium only slightly perturb the $1.2 \mathrm{~A}$ long $\mathrm{C}-\mathrm{H}$ bond that is oriented, in equilibrium, along the $\mathrm{Z}$ axis. Conservatively, we will ignore these in plane oscillations.

In the harmonic approximation to the $\mathrm{C}-\mathrm{H}$ bond about its minimum, the ZPM of the carbon atom lying at the other end of "the spring" that attached to the ${ }^{3} \mathrm{H}$ atom is formed by a superposition of many frequencies. The impurity tritium atom may further give rise to localized phonon modes. Oscillatory perturbing forces acting on the ${ }^{3} \mathrm{H}$ atom that are associated with random relative phases will add up in quadrature. The 4 times heavier carbon implies that the average squared vertical velocities $\left\langle V_{Z}^{2}\right\rangle$ of the carbon atoms is only $1 / 4$ of the average squared vertical velocities $\left\langle V_{Z}^{2}\right\rangle$ of the tritium relative to the bonding carbon. With the velocities adding in quadrature, we therefore expect that the extra $\Delta V_{z}$ and associated blurring by $\Delta E$ will increase only by $\sqrt{1+(1 / 4)}-1 \sim 11.8 \%$ relative to the ZPM effect obtained in the earlier sections without considering the phonons.

\section{TWO EXAMPLES}

We next recall two examples from high energy physics of broadening due to "zero point" internal motion of electrons in atoms and of nucleons in the nucleus.

Glashow process. The first of these two examples is the Glashow process, where a $\bar{\nu}_{e}$, most likely of extragalactic origin, of an extremely large energy,

$$
E\left(\bar{\nu}_{e}\right)=E(\operatorname{Res})=\frac{M(W)^{2}}{2 m(e)} \sim 6.4 \times 10^{15} \mathrm{eV},
$$

interacts with terrestrial atomic electrons producing the mediator of weak interactions, the $\mathrm{W}$ boson, of mass $M(W) \sim 80 \mathrm{GeV}$, and width $\Gamma(W) \sim 2 \mathrm{GeV}$. The rate of the production of $W^{-}$in the resonant region, where

$$
E\left(\bar{\nu}_{e}\right)=E(\operatorname{Res})\left(1 \pm \frac{\Gamma(W)}{M(W)}\right)
$$

and even in a much broader region, is very large, exceeding the reaction rate of the other five neutrino species combined. Thus, despite the paucity of such high energy neutrinos, some events in the large Ice-Cube detector were expected, and most recently, a clear cut event has been detected [14]. Glashow [15] noted that ZPM of atomic electrons broadens the resonance by as much as a factor of 2 . This has the amusing and potentially observable consequence of doubling the $\sim 10 \mathrm{Km}$ mean free path of Glashow neutrinos in the Earth [16]. The few eV kinetic energy of the outgoing ${ }^{3} \mathrm{He}^{+}$in tritium decays is $\sim 10^{-15}$ times smaller than the neutrino energy in the Glashow process, indicating a remarkably wide range over which the smearing via zero point motion applies.

Nuclear Targets. The second, better known, example involves nuclear targets. The internal Fermi motion of the nucleons in the nucleus is enhanced by the exclusion principle and the velocities of these nucleons can reach $\sim 0.3 c$. The resulting shifts of invariant masses of a high energy projectile and such a nucleon can lower by $30 \%$ the minimal energy required for producing a particle $X$, or a pair $\bar{X}-X$, relative to the threshold for the same reaction on a free nucleon target. An analog of this in the present case is that, when the ZPM aligns with $\vec{p}(e)$, the momentum of the emitted $\beta$ electron the latter has an energy exceeding the "kinematic bound" of $Q+2 m(\nu)$. Unfortunately, this "good effect" is offset and reversed by the upward shift of the energies of the many electrons in the steeply falling continuum spectrum just below $Q$.

There is a qualitative difference between the above two high energy reactions where both incoming and outgoing 
particles are (ultra)relativistic and the present case where the outgoing ${ }^{3} \mathrm{He}^{+}$or ${ }^{3} \mathrm{He}$ atom recoils with a velocity: $V=$ $p(\beta(e l)) / M\left({ }^{3} \mathrm{He}\right)$ of $\sim 3 \times 10^{-5} c$. One may then wonder if, for a graphene substrate, we have to account for the fact that the new potential(s) $\mathcal{V}^{\prime}$ on the ${ }^{3} \mathrm{He}^{+}$ion (or the ${ }^{3} \mathrm{He}$ atom) may slow down or even stop their exit from the graphene sheet an issue that we will address in detail later. This highlights the fact that, thus far, only the lowest bound state in the initial potential $\mathcal{V}=\mathcal{V}\left({ }^{3} \mathrm{H}\right.$-graphene $)$ was used with no direct reference to this or the new potential $\mathcal{V}^{\prime}=$ $\mathcal{V}^{\prime}\left({ }^{3} \mathrm{He}^{+}\right.$-graphene) following the $\beta$ process (or the one between the graphene and a final helium atom). A main objective in this work is to rectify this shortcoming and investigate the effect of the graphene dynamics - specifically of its electronic excitations - on the blurring. Indeed, the electrons in the graphene moving with velocities that at at the top of the valence band reach a (Fermi) velocity $v_{F}=3 \times$ $10^{-3} c$ generate the potentials between the initial tritium or its helium ion/atom predecessors and the graphene.

We next turn to a main motif of this paper-that of viewing all smearing effects as due to the sudden change of the Hamiltonian describing the host system.

\section{SMEARING VIA SUDDEN CHANGES OF THE HOST SYSTEM}

Much insight into numerous problems is provided in situations when a hierarchy of timescales exists. The shortest here is the time it takes the $\beta$ electron to escape from the residual ${ }^{3} \mathrm{He}^{+}$,

$$
\delta_{\beta \text {-escape }}(t) \sim \frac{a_{\mathrm{Bohr}}}{v(\beta(e))} \sim 10^{-18} \mathrm{sec} .
$$

In the above, $v(\beta(e))$ is the velocity of the $\beta$ electrons of energy close to the end point $\epsilon(e) \sim Q=18.6 \mathrm{KeV}$,

$$
v(\beta(e))=\frac{p(e)}{m(e)}=[2 Q / m(e)]^{1 / 2} \sim 0.26 c,
$$

and $a_{\mathrm{Bohr}} \sim 0.55 \mathrm{~A}$ is the Bohr radius. The velocity $v(\beta(e))$ exceeds the relevant velocities of the hydrogen atomic electron $\sim c / 137$ by a factor of $\sim 0.26 \times 137 \sim 35$.

Thus, the escape of the $\beta$ electron causes a sudden change of the Hamiltonian describing the various hosts of the decaying tritium nucleus. As we discussed earlier, these hosts may be (a) atomic, (b) molecular if the original ${ }^{3} \mathrm{H}$ is a member of a ${ }^{3} \mathrm{H}-{ }^{3} \mathrm{H}$ molecule, or (c) a solid as in, e.g., the ${ }^{3} \mathrm{H}$ atom absorbed on a graphene sheet. Specifically, in the latter, the initial Hamiltonian for the host system with the tritium atom bound to the graphene transforms at $t=0$ (within the above short $\delta t$ time interval) to the new Hamiltonian appropriate for the same graphene sheet with the tritium $Z=1$ nucleus replaced by the helium $Z=2$ nucleus at the same initial location.

In the sudden approximation which applies for all the above hosts, the time dependent Hamiltonian interpolating between the initial and final Hamiltonians acts for too short a time and hence, does not change the global initial wave function $\left|\Psi_{i}\right\rangle$ of the host system (defined to be the complete initial system less the escaping $\beta$ electron). The energy of the host does however change in the sense that

$$
\Delta E=\left\langle\Psi_{i}\left|\mathcal{H}^{\prime}\right| \Psi_{i}\right\rangle-\left\langle\Psi_{i}|\mathcal{H}| \Psi_{i}\right\rangle=E^{\prime}-E_{0} \neq 0 .
$$

Here, $\mathcal{H}$ and $\mathcal{H}^{\prime}$ denote, respectively, the global system Hamiltonians before and after the $\beta$ process (these Hamiltonians contain the potential energy contributions $\mathcal{V}$ and $\mathcal{V}^{\prime}$ discussed above). In Eq. (25), we used the fact that the initial low temperature state was very close the ground state of the original Hamiltonian $\mathcal{H}$. An energy change of the environment is indeed expected here as in all of the cases with a time dependent potential. Energy can then be exchanged with the external "agent," which provides the time dependent potential - the role of which is played here by the escaping $\beta$ electron-whose energy far exceeds the atomic scale energies of $\sim 10 \mathrm{eV}$, which are relevant to the problem at hand.

In the eigenbasis of the new Hamiltonian, the standard time evolution of the initial state is

$$
\begin{aligned}
|\Psi(t)\rangle & =e^{-i \mathcal{H}^{\prime} t / \hbar}\left|\Psi_{i}\right\rangle \\
& =\sum_{n} e^{-i E_{n}^{\prime} t / \hbar}\left|\Psi_{n}^{\prime}\right\rangle\left\langle\Psi_{n}^{\prime} \mid \Psi_{i}\right\rangle .
\end{aligned}
$$

The probability $P(i \rightarrow f)=\left|\left\langle\Psi_{i} \mid \Psi_{f}^{\prime}\right\rangle\right|^{2}$ of winding up in any specific final state $\left|\Psi_{f}^{\prime}\right\rangle$ satisfies unitarity, $\sum_{n} P_{i \rightarrow n}=$ $\sum_{n} P_{n}=1$, and the total energy shift condition $\sum_{n} P_{n} E_{n}=$ $E_{0}+\Delta E$. For times exceeding the escape time of the $\beta$ electron, we may neglect the interactions between the exiting $\beta$ electron and the remaining host system and the conservation of the overall energy $\epsilon(\beta(e))+E_{n}^{\prime}$ (Host) "entangles" the energy of the escaping $\beta$ electron with the energy of the final state that the host winds in. At times larger than the inverse of the energy splitting, the different states tend to "decohere" [17] and the system "settles" into one of the new energy eigenstates (with the emitted $\beta$ electron occupying a continuum state of complementary energy). This decoherence is related to the time-energy uncertainty relation $\Delta E \times \Delta t \geq \hbar / 2$, which implies that at short times, after the transition to the new Hamiltonian, the energy of the atomic electron is not well defined. This also holds for the out-going $\beta$ electron since energy conservation entangles it with the atomic system so that both have the same spread $\Delta E=\delta \epsilon(e))$. As time goes on, these energies can get sharper as $\sim 1 / t$, and smaller variations of levels of the host system get imprinted on the energy distribution $F(\epsilon(\beta(e)))$ of the final $\beta$ electrons.

While the $\beta$ electrons travel through the spectrometer towards the detector, many things can happen to the host system. However, in general, these developments do not affect the energy of the $\beta$ electrons and can be ignored in estimates of the smearing. This holds for any process where 
energy is exchanged between different parts of the host system leaving its total energy, and hence, also that of the escaping $\beta$ electron, unchanged. An example is afforded by the decay of the excited $n=2$ final state of the hydrogen-like $\mathrm{Z}=2{ }^{3} \mathrm{He}^{+}$to the $n=1$ ground state and photons. The key observation is that the electromagnetic field with which the ${ }^{3} \mathrm{He}^{+}$ion shares its energy, makes, together with the ${ }^{3} \mathrm{He}^{+}$ion, the extended "environment" hosting the initial tritium nucleus.

Another example where this feature greatly helps estimate the magnitude of the blurring effect is that the final ${ }^{3} \mathrm{He}^{+}$ion and/or the ${ }^{3} \mathrm{H}$ atom, may, in experiments using graphene, excite various degrees of freedom in the graphene lattice. However, both the graphene and the atom, in which the initial radioactive nucleus resided, are parts of the extended environment and energy transfers between the translational motion of the recoiling ion/atom and the graphene will_ just like the radiation of photons in the above example, not change the energy of the environment - nor that of the beta electron.

In the case of molecular tritium, one can, in principle, directly measure the various final states of, say, the helium hydride ion ${ }^{3} \mathrm{H}_{-}^{+}{ }^{3} \mathrm{He}$ by tagging it in coincidence with the measured $\beta$ electron. Indeed, the probability of having various states the helium hydride ion was measured by tagging the recoiling hydride and the infrared photon emitted when the hydride deexcites from some level to another. Such tagging could help mitigate difficulties due to smearing. Unfortunately, it is completely impractical in the experiments of interest. It takes $\sim 10$ microseconds-millisecond for the radiative deexcitation of the helium hydride ion and/or for the hydride to travel to the detector. To enable tagging, there should be no more than one coincident $\beta$ electron emitted in this time interval. This allows for, at most, $3 \times 10^{12} \beta$ electrons to be collected in a year. As may be inferred from Fig. 1 and the full spectral shape of the tritium $\beta$ decay, this is far less than what is required in order to produce the extremely rare $\mathrm{CNB}$ capture events. We have no independent information on the final energy eigenstate $\left|\Psi_{n}^{\prime}\right\rangle$ that the host winds up in, apart from its energy $E_{n}^{\prime}$ [inferred from $\epsilon(\beta(e))$ ].

A key point is that only the cases where after the exit of the $\beta$ electron the system hosting the tritium lands in, or very near to, the lowest possible energy state(s) are relevant. This is so because by energy conservation the outgoing $\beta$ electrons then have the maximal energy possible, and only such events can contribute to searches of light mass and/or the CNB neutrinos by investigating the spectrum of the $\beta$ electrons at and above the end point.

An immediate consequence of all of the above is that we should consider only the cases when the ${ }^{3} \mathrm{He}^{+}$ion remaining after the escape of the $\beta$ electron is in its ground state. Also, whenever the tightly bound ${ }^{3} \mathrm{He}$ atom can form, we should focus on the "branch" where it did form and is, furthermore, in the atomic ground state. If possible, this helium atom should be bound to the rest of the host- the other hydrogen atom in the molecular case or to the embedding graphene. Finally, this last bound state should be in, or near, its ground state.

To have these peak energy electrons clearly stand out and be separated from the lower energy crowd, the hosts of the radioactive tritium atoms should satisfy the following two conditions:

(i) Condition $\mathrm{A}$

There is a substantial overlap of the initial state of the host and the lowest energy eigenstate $\left|\Psi_{o}^{\prime}\right\rangle$ of the final system after the emission of the $\beta$ electron [18]. This single state may be replaced by a group of final states with energies in a $\Delta$ neighborhood of the lowest energy $E_{\min }^{\prime}$, which jointly have a large overlap with the initial state of the host. Specifically, the sum over these final states $\sum_{n}\left|\left\langle\Psi_{n}^{\prime} \mid \Psi_{i}\right\rangle\right|^{2}$ should constitute an appreciable fraction of the complete sum over all states which by unitarity is equal to one. This ensures that in a substantial fraction of all events, the host winds up with energies $\left(E_{\min }^{\prime}+\Delta\right) \geq E_{n}^{\prime} \geq E_{\min }^{\prime}$.

To avoid the extra quantum mechanical smearing from exceeding the experimental resolution in the measurements of the $\beta$ electron energies $\delta(\epsilon)_{\exp }$, we take $\Delta$ to be equal to the latter. The CNB peaks generated by electrons associated with the above states, could still be "swamped" by neighboring, slightly lower energy, electrons associated with other final states lying above but close to $E_{\min }$ which collectively form with probability higher than that of the above "good" group of states in condition A. To avoid this we need to satisfy a second condition.

(ii) Condition B:

The lowest state (or set of lowest states defined in condition A above) is well separated from the next higher energy state (or set of states), which are generated with a higher probability, by an energy gap larger than $\sim 4 \Delta$.

We next turn to the well-studied cases of atomic [19] and molecular hosts [1]. These provide concrete and rather instructive examples of the above general discussion of smearing within the overarching framework of spectra of states near the lowest energy generated by the sudden escape of the $\beta$ electron.

\section{ATOMIC AND MOLECULAR HOSTS}

The effect of changing potentials on the atomic electron was referred to above as the $\delta E_{\text {atomic }}$ term. The $Z=1$ Coulomb potential $\mathcal{V}$ in the tritium becomes, after the $\beta$ electron is emitted, a new potential $\mathcal{V}^{\prime}$-the $Z=2$ Coulomb potential of the ${ }^{3} \mathrm{He}$ nucleus. Along with the wave function, the kinetic energy of the electron remains the same but the potential attraction is doubled so that the overall energy change in this case is $-e^{2} / a_{\mathrm{Bohr}}=-2 R y$. Using the hydrogenic $1 \mathrm{~S}$ wave functions with $\mathrm{Z}=1$ and $\mathrm{Z}=2$ for 
the initial and final states, $\Psi_{i}$ and $\Psi_{f}^{\prime}$, respectively, we find that in the sudden approximation the probability that the host system will stay in the new ground state is

$$
\left|\left\langle\Psi_{Z=1} \mid \Psi_{Z=2}^{\prime}\right\rangle\right|^{2}=\frac{2^{9}}{3^{6}} \sim 0.64
$$

The fact that the overlap with the new ground state is a substantial fraction means that condition $\mathrm{A}$ is well satisfied here. The spacing of $3 R y \sim 41 \mathrm{eV}$ between the $1 \mathrm{~S}$ ground state and the first excited $2 \mathrm{~S}$ state of ${ }^{3} \mathrm{He}^{+}$(a state which in any event is produced with less than half the probability of the ground state) is indeed very large. It is 400 times bigger than $\Delta$, which we take equal to a resolution goal of $0.1 \mathrm{eV}$ so that condition B is most amply satisfied.

This seems to make atomic tritium with no intrinsic spreading, an ideal candidate for sensitive searches for neutrinos. Unfortunately, it is very difficult to prevent cold atomic hydrogen from converting into its molecular form. We thus next turn to the case when the tritium is part of a diatomic ( ${ }^{3} \mathrm{H}-$ - $\mathrm{H}$ ") molecule, where " $\mathrm{H}$ " denotes a hydrogen or its D or T isotopes. We then need to consider the new molecular states appearing after the sudden escape of the $\beta$ electron.

A notable difference from the atomic realization above, where only one atomic electron is inherited from the original ${ }^{3} \mathrm{H}$ atom, is that in the molecular case there are two electrons in the immediate ( $\sim$ angstrom) vicinity of the helium nucleus. Both electrons can now wind up in the $1 \mathrm{~S}$ shell of helium forming a final neutral ${ }^{3} \mathrm{He}$ atom in its ground state. The first ionization energy of $\mathrm{He}$ is higher than that of hydrogen by $\sim 12 \mathrm{eV}$. This endows the $\beta$ electrons entangled with the final state containing the helium atom with energies which are $\sim 12 \mathrm{eV}$ higher as compared with the atomic case above where only a single electron winds up in the $1 \mathrm{~S}$ state in ${ }^{3} \mathrm{He}^{+}$. Again, only these highest energy $\beta$ electrons make the highest end point and putative CNB peak(s) beyond it thereby contributing to the experimental search. All other $\beta$ electrons emitted in conjunction with higher final molecular states and/or ionized helium will have lower energies and will not play any role in tritium end point experiments with sub-eV neutrinos. The probability that the $\mathrm{He}$ atom will form is given by the overlap of the initial state where the two electrons make the covalent $\mathrm{H}-\mathrm{H}$ bond and the ground state of the ground state of para-helium. While the fact that both in the initial and final state the spins of the two electrons are coupled to a total spin $S=0$ clearly enhances the probability $\mathrm{P}(\mathrm{He})$ of the branch where an atomic para-helium is formed, the fact that here a pair of electrons (instead of a single electron) need to overlap, suggests that $\mathrm{P}(\mathrm{He})$ is smaller than the probability that we obtain the ground state helium ion in the atomic case of 0.6.

The final helium in its ground state can still bind with the ionized " $\mathrm{H}$ "+, namely the proton or the deuteron or tritium nuclei. The polarization by a pointlike, charged " $\mathrm{H}$ "+ nucleus generates an attractive potential, which at large distances is [20]

$$
V=-\frac{\alpha_{e m} \alpha_{\mathrm{He}}}{2 R^{4}},
$$

with $\alpha_{\mathrm{He}}$ the electric polarizability of helium (and $\alpha_{e m}$ the fine structure constant). With no Pauli exclusion to prevent the bare, positively charged nucleus from penetrating the electron cloud in the helium, a significant attractive potential which extends to small distances, is being generated. This potential then tightly binds the helium atom and the hydrogen nucleus the helium hydride ion: " $\mathrm{H}$ "+3 He with binding $\mathrm{B} \sim 1.68 \mathrm{eV}$ and average radius of $\sim 0.8$ A [21].

The maximal reduced mass $\mu\left({ }^{3} \mathrm{H}^{+}{ }^{3} \mathrm{He}\right) \sim 3 M(H) / 2$ maximizes the binding and minimizes the recoil energy of the hydride in the case of an initial diatomic tritium molecule. This elevates the end point of the associated $\beta$ spectrum and the putative CNB capture peaks above the corresponding end points and peaks in the decays in TD or TH molecules-ameliorating the effect of a possible small admixture of molecules with the lower mass $\mathrm{H}$ isotopes. Choosing " $\mathrm{H} "=T$ is also preferred experimentally because it doubles the amount of radioactive tritium, and we will focus on this case.

Calculations of the collective overlap of the initial, post $\beta$ reaction, state, and the ground or excited states of the final ${ }^{3} \mathrm{He}-\mathrm{T}$ suggested very high values of $\mathrm{P}=0.93$, [22] These values strongly conflicted with experimental measurements, which found a probability of $P \sim 0.5$ - an embarrassing state of affairs which lasted until 1989 when the important paper [1] rectified it. A clear review, of both the experimental and theoretical status, and much original work is contained in the 2015 Ph.D. thesis of Laura I. Bodine [2].

The significant overlap with the cluster of states within $\sim 0.1 \mathrm{eV}$ above the ground state of ${ }^{3} \mathrm{He}-\mathrm{T}^{+}$implies that condition A above is satisfied. To check if conditions $\mathrm{B}$ also holds, we need to find the behavior of the $E_{n}^{\prime}$ energy levels of the helium hydride ion. A study of these states, far more detailed than what we present next, is included in [1], in the above noted thesis of Laura Bodine [2], and in many yet more recent theoretical works. The following somewhat heuristic approach may still be useful. The lowest excitations are rotational and the energy of levels of angular momentum $l$ are

$$
E_{l}=\frac{\hbar^{2} l(l+1)}{2 \mu R^{2}} \sim l(l+1)(1.2) \mathrm{meV},
$$

where $\mu=M(T) / 2=3 M(H) / 2$ is the reduced mass, and a molecular radius $R$ of $\sim 1$ A was used. The maximal $l$, fixed by the total binding in this simplistic approach, is 
$l_{\max }=L \sim 40$. Both $l$ and $l_{Z}$ (the projection along the $\vec{R}_{1}-$ $\vec{R}_{2}$ symmetry axis of the molecule) depend on the uniformly distributed direction of the momentum of the $\beta$ electron relative to this axis. For each $l$, there are $2 l+1$ states of varying $l_{z}$ values. Thus, between $l=0$ and a maximal $l=L \sim 40$, there are altogether $\sum_{l=0}^{L}(2 l+1)=$ $L^{2} \sim 1600$ rotational states of energies extending up to the binding energy of the helium hydride ion of $1.7 \mathrm{eV}$ with the same average small separation of $1 \mathrm{meV}$ between consecutive levels. Also, in a simple classical picture used above, justified in retrospect for the large $l$ values, the probabilities of having the different levels are the same.

The above discussion focusing solely on the rotational modes suggests that the $1.7 \mathrm{eV}$ interval above the lowest energy final state hydride is uniformly populated by a dense set of helium ion bound states, so that condition B above may still be satisfied. However, at higher energies, the vibrational excitations and their associated rotation bands restore the well known general tendency of energy spectra to get denser at higher energies. This, in turn, leads to a violation of condition $\mathrm{B}$ and to appreciable smearing.

Furthermore, the higher vibrational modes that may nearly tear the molecules apart smoothly connect the bound hydride ion and its dissociated form of ${ }^{3} \mathrm{He}$ and $T^{+}$. We consider the latter next. In such decays to three on shell particles,

$$
\nu(1)+\left({ }^{3} \mathrm{H}^{3} \mathrm{H}\right) \rightarrow e^{-}+{ }^{3} \mathrm{H}^{+}+{ }^{3} \mathrm{He},
$$

the final energies are not sharp. Momentum conservation implies only that the momenta of the final particles lie in a plane and the total kinetic energy $E=E\left({ }^{3} T^{+}\right)+E\left({ }^{3} \mathrm{He}\right)$ of the recoiling atom and ion depends on the angles between the momenta in this plane. These angles vary between different events causing a spread $\Delta E$ of the energy of the heavy particles. Kinematics alone allows $\Delta E$ to extend over an interval of size $\Delta E \sim E(\max )-E(\min )=E_{\text {free }}-\frac{E_{\text {free }}}{2}=$ $\frac{E_{\text {free }}}{2} \sim 1.85 \mathrm{eV}$. Here, $E(\mathrm{~min})$ is the minimal total recoil state of interest, corresponding to equal momenta of ${ }^{3} \mathrm{H}^{+}$and of ${ }^{3} \mathrm{He}$ so that the $\beta$ electron effectively recoils against a system of mass $2 M=6 M(H)$. The dynamics prescribing the probabilities of final states with different recoil energies is encoded in the momentum space wave function of the ground state of the initial T-T molecular analog of $\tilde{\psi}_{0}(\vec{K})$ mentioned above in connection with smearing due to ZPM of the tritium atom relative to the graphene. We have noted that this ZPM effect can be viewed as part of the general "host connected broadening" framework. This clearly applies to the molecular case. The spreading due to the different recoil energies of the ${ }^{3} \mathrm{He}$ atom - the descendant of the original tritium - is in fact the very same spreading induced by the zero point vibration of this tritium atom and fully accounted for by the latter.

The $\mathrm{H}-\mathrm{H}$ bond is $\sim 2.5$ times stronger than the $\mathrm{C}-\mathrm{H}$ chemisorption potential used in estimating the zero point smearing effect for tritium adsorbed on graphene, and the reduced mass here is $5 / 8$ times smaller. This renders the ZPM blurring effect here twice as large $\sim 0.48 \mathrm{eV}$. This, however, may not be extremely important as (i) these dissociated states lie above the lower energy bound states of the helium hydride ion and (ii) kinematics implies that dissociation does not occur in most cases.

In principle, an exhaustive calculation can be done (and in view of the big experimental effort is indeed ongoing). This requires detailed computations of the wave functions and energies of the rotational, vibrational levels of the ${ }^{3} \mathrm{He}-$ " $\mathrm{H}$ " molecule-ion, and the overlaps of these states with the state $\Psi_{i}$ generated immediately after the $\beta$ process happened at $t=0$. The locations $\vec{R}_{1}$ of the newly formed ${ }^{3} \mathrm{He}$ and $\vec{R}_{2}$ of the other "H" are the same as those of the two atoms in the ${ }^{3} \mathrm{H}$-" $\mathrm{H}$ " molecule and the ${ }^{3} \mathrm{He}$ has the momentum kick $\vec{P}=-\vec{p}(\beta(e))$. In real space, this wave function assumes the form,

$$
\left\langle\Psi_{i} \mid \vec{R}_{1}, \vec{R}_{2}\right\rangle=e^{i \vec{P} \cdot \vec{R}_{1} / \hbar} \Psi_{{ }^{\mathrm{H}-“} \mathrm{H}^{\prime}}\left(\vec{R}_{1}-\vec{R}_{2}\right),
$$

where $\Psi_{{ }^{H}-\text { " } \mathrm{H}}$, is the initial wave function of the $\mathrm{H}-$ " $\mathrm{H}$ " molecule in its ground state described in terms of the $\mathrm{H}-$ " $\mathrm{H}$ " relative separation $\vec{R}=\vec{R}_{1}-\vec{R}_{2}$. The factor $\exp \left[i \vec{P} \cdot \vec{R}_{1} / \hbar\right]$ "imparts" a recoil momentum $\vec{P}$ at the location of the ${ }^{3} \mathrm{H}$ atom.

Presently, the KATRIN experiment using molecular $T_{2}$ is hampered by experimental difficulties. A recent publication [23] presenting KARTIN's latest upper bound on the mass of the lightest neutrino of $1.1 \mathrm{eV}$, listed the many experimental issues that prevented reaching the initial, much more ambitious, goal of KATRIN.

\section{GRAPHENE REVISITED}

Using tritium stored inside solid materials and/or adsorbed on surfaces may reduce the experimental difficulties associated with monitoring large quantities of gaseous tritium by using large $\beta$ spectrometers. In a most optimistic scenario, the experimental technique may improve to the point of allowing searches for light CNB. In this case, a careful study of the irreducible quantum noise in the new proposed experiments is of paramount importance. We continue to do so here for the "benchmark" case of tritium adsorbed on a graphene sheet.

Our discussion of the molecular tritium decays showed that adding one extra particle to the tritium atom generated significant smearing thanks to the dense spectrum of final ${ }^{3} \mathrm{He}-$ "H" states. As we argue in this section, the much more complex excitations spectrum of the many body graphene system yields much stronger smearing when the tritium is adsorbed onto graphene.

The smearing effects fall into two categories: (i) blurring due to nuclear motions and those due to (ii) electronic excitations. The overall scale of electronic energies is 
higher than that of the nuclear vibrational/ rotational modes. Still, all smearing encountered above were due to the dense spectrum of the latter. Thus, the large dispersion by more than $\sim 40 \mathrm{eV}$ of the energy spectrum of the final helium ion caused no harmful smearing in the case of atomic tritium decays because of the extremely sparse nature of this spectrum. The graphene has a similar large span of a $\sim 15 \mathrm{eV}$ broad valence band. However, the energy spectrum in this band is continuous and dense suggesting extensive smearing.

Can we approach this problem in a more quantitative fashion? The rapid escape of the $\beta$ electron justifies using the sudden approximation. In this approximation, we have at $t=\delta t(\beta$ escape $)$, the $Z=2$ helium nucleus, endowed with the free recoil energy sitting at $\vec{R}=\left(0,0, Z_{0}\right)$ the original location of the parent tritium which is shifted relative to its binding carbon atom at the origin by $\sim Z_{0}=$ $1.2 \mathrm{~A}$. To further justify the sudden approximation and better realize the relatively large number of electrons involved, we note the following: For a distance $\rho$ from the location at $\left(0,0, Z_{0}\right)$ of the initial tritium, it takes a time of only $\rho / c$ to establish the new potential generated after the escape of the negative $\beta$ electron. This region contains $N_{C}=\pi(c t)^{2} \times$ the number density of carbon atoms in graphene (of $3.82 \times 10^{15} \mathrm{~cm}^{-2}$ atoms). There is only one free electron per carbon as three out of the four outer shell electrons form $s p^{2}$ hybridized covalent bonds with the three neighboring carbons. Thus, the same number of $N_{e}=$ $N_{C}$ electrons will be influenced. The final helium nucleus moves very slowly with velocity $V \sim 3 \times 10^{-5} \mathrm{c}$. Using the size of the graphene unit cell, we find that by the time it moved just $0.1 \mathrm{~A}, N_{C} \sim 0.9 \times 10^{7}$ carbon atoms and an equal number of electrons are affected. For the purpose of our qualitative discussion, the number of electrons that can reach the origin in this time interval may serve as a measure of the complexity actually encountered. Using electron velocities of the order of the Fermi velocity $v_{\text {electron }} \sim v_{F}=3 \times 10^{-3} c$, which is appropriate near the top of the valence band, we then find that "only" $\left(v_{F} / c\right)^{2} N_{c} \sim 100$ electrons are likely to be engaged. For a complete calculation, we need to find the probabilities for "exciting" (in the sudden approximation) any one of the many levels of the graphene in either one of the two main "branches" where the helium ion ${ }^{3} \mathrm{He}^{+}$or the helium atom ${ }^{3} \mathrm{He}$ has formed. In principle, this requires the full wave function of the initial ground state of the graphene $N$-body system with the bound tritium impurity at $\vec{R}=$ $\left(0,0, Z_{0}\right)$. Next, we need to compute the wave functions and energies of the states arising when the $Z=1$ tritium nucleus is replaced, after the escape of the $\beta$ electron, by the $Z=2$ helium nucleus. Finally, we must compute the overlaps of the initial state with each of these many final energy eigenstates so as to produce the spectrum of excitations. At first sight, such an undertaking seems impossible. While the ground state of perfect graphene is (almost) fully understood, the study described above requires extensive calculations. The simplifying concepts of conduction/valence bands rely on the invariance with respect to translation by lattice vectors, an invariance that is maximally broken near the impurities. However, the very fact that the initial and final Hamiltonians $\mathcal{H}$ and $\mathcal{H}^{\prime}$ differ only locally, in the region near the "impurities," is of great help. It guarantees that the overall energy change induced by the sudden $\beta$ process $\Delta E$ will be $\leq 10 \mathrm{eV}$, and that only a limited number of electrons could be affected.

We can further take advantage of this locality by using a finite grid to simulate the two-dimensional graphene lattice around the impurity of interest. As an example, we recall the well studied case [24] of the electron-hole exciton in graphene, where such $a b$ initio calculations correctly predicted the exciton energy levels and response to radiation.

Equally important is the fact that in order to estimate the spreading near the end point, we do not need the complete excitation energy spectrum-but only in the vicinity of the new ground state. As emphasized, this ground state is in the "helium branch" of the final states which contain helium in its ground state. Hopefully, this branch has a significant weight as otherwise investigation of the end point and vicinity will be plagued by smearing and by a reduced statistics.

The relatively deeply bound (by $1.7 \mathrm{eV}$ ) helium hydride ion plays an important role in experiments involving molecular tritium. Naturally, one may wonder how strongly bound is the helium atom to the rest of the system (that, in the present context, is the entire graphene sheet). Note that because the electron mass is 3000 times smaller than the reduced mass in the case of the helium hydride, there is, most likely, no bound state of helium and a free electron. However, the electrons inside the graphene-and, in the present situation, the hole, which the escape of the $\beta$ electron created, are strongly bound to the graphene so as to effectively carry all of its inertia. We will not attempt here to calculate this binding beyond noting that it is likely to be stronger than that of the helium hydride ion. The reduced mass here is $\mu=$ $\mathrm{M}\left({ }^{3} \mathrm{He}\right)=3 \mathrm{M}(\mathrm{H})$ instead of $\mu=3 M(H) / 2$. The invariance with respect to shifts of the helium atom parallel to the $(\mathrm{XY})$ plane of the graphene sheet suggests that the problem effectively become one dimensional. The long range $R^{-4}$ attractive potential between the helium and a pointlike charge [20] becomes $Z^{-4}$ with the same coefficient, and with the kinetic terms associated with momenta in the XY plane omitted, the kinetic energy is further reduced by $\sim 66 \%$.

Let us assume that future calculations will reveal that (a) The helium is indeed bound to the graphene sheet with a very large binding energy and that as for the helium hydride ion in the molecular tritium case there is a considerable overlap with this particular state yet (b) unlike for the above helium hydride, there are no such important excited graphene-helium states.

In analogy with the discussion of atomic tritium, we may then expect no smearing. Unfortunately, this is not the situation here as there is a continuum of graphene 
electronic excitations. Specifically, this amounts to saying that assumption (b) is untenable. Even if the potential model and the motion of the bound tritium alone do not lead to a dense excitation spectrum, the electronic excitations of the graphene may do so.

We next present a simple estimate of the smearing due to the electronic excitations in the graphene. We saw above that up to 90 electrons can reach the origin-i.e., the location of the carbon atom situated just below the original adsorbed ${ }^{3} \mathrm{H}$ atom, while the final ${ }^{3} \mathrm{He}^{+}$or ${ }^{3} \mathrm{He}$ move a distance of just 0.1 A. Only a small fraction of these electrons actually "move" to the immediate vicinity of the origin-defined here as the unit cell around the binding carbon at $\vec{R}(t=0)=(0,0,0)$ as their migration is blocked by both the Pauli exclusion and by mutual Coulomb repulsion. Only one unit of negative charge is needed in order to neutralize the positive charge generated by the sudden escape of the $\beta$ electron. This single extra electron can affect the formation of the neutral final helium atomthe branch that we focus on. As the relevant electrons are initially localized within distances of $\Delta r \sim 1-3$ A, away from the $\beta$ decay event, their momentum spread is $\hbar c \Delta k \sim \frac{\hbar c}{2 \Delta r} \sim 0.33-1 \mathrm{KeV}$. Given that $\Delta r$ is similar to the size of the lattice unit cell, such a momentum spread, close to the energy scale of the Brillouin zone, is indeed expected. Electrons shifted in energy by less than $\Delta E^{\prime}=$ $1 \mathrm{eV}$ from the Dirac point obey a linear dispersion rule $E(k)=\hbar v_{F} k$. Substituting the graphene Fermi velocity $v_{F}=3 \times 10^{-3} c$ yields a spreading of energies of size,

$$
\Delta E \sim \hbar v_{F} \Delta k=\frac{\hbar v_{F}}{2 \Delta r} \sim 1-3 \mathrm{eV} .
$$

The above rather heuristic arguments do not replace a direct evaluation of the overlaps between the initial state shortly after the escape of the $\beta$ electron with the lowest energy cluster of final eigenstates of the new Hamiltonian $\mathcal{H}^{\prime}$. However, finding which electrons in the graphene are "mobilized" by the new Hamiltonian helps estimating the expected smearing. This type of information is encoded in the time evolution $|\Psi(t)\rangle=e^{-i \mathcal{H}^{\prime} t / \hbar}\left|\Psi_{i}\right\rangle$ of the initial wave function under the new Hamiltonian when expressed in configuration space rather than in terms of the new energy eigenstates. While this does not achieve the required projection onto the subspace of the lowest energy states, it gives us an idea of the overall energy shift induced by the $\beta$ process. The claimed smearing then follows if this total shift is of the order of an $\mathrm{eV}$ and no prominent low energy state can account for this energy change as was the case for atomic tritium decays. Finally, the dual use above of both momentum and coordinate space is naturally called for by the problem at hand with the extended lattice and its bands on the one hand and the local impurity at the origin on the other.

A slightly different, more succinct, way of rephrasing the argument is the following: The important change instigated by the escape of the $\beta$ electron is that the region near to the original tritium becomes positively charged. One can therefore expect significant overlap with new energy eigenstates, where negative charges moved towards the origin in order to neutralize this positive charge. The fact that the holes generated in this process are produced at various depths in the band means that the graphene system will have this spread of energies which, in turn, will be reflected in a substantial smearing of the $\beta$ electrons energy distribution $F(\epsilon(\beta(e)))$. The lowest energy holes are at the Dirac point at the tip of the cone at the top of the valence band. This single point has zero measure. However, the area of the intersection of the conical valence band and fixed energy planes increases quadratically with the (absolute) value of the energy of the hole like $E(h)^{2}$ as we go deeper into the band. This, then, causes a smearing which, in principle, could be as large as the full width of the valence band of $\sim 15 \mathrm{eV}$.

So far, we have largely ignored the branch with the helium ion ${ }^{3} \mathrm{He}^{+}$in the final state as the energies in this branch are upward shifted relative to the helium atom branch by $\sim 12 \mathrm{eV}$. In the remainder of this section, we will address this important branch. This is required in order to gain a better picture of the overall $\beta$ electron spectrum, also away from the immediate vicinity of the end point. A first observation is that the arguments re overlaps of the "initial" state, formed early on, at times $\sim \Delta t_{\beta(e) \text { escape }}$ before the branching into the helium atom or helium ion sector occurred, equally apply to the helium ion branch.

The momentum of the heavy descendant of the initial tritium, be it the helium ion or the helium atom is large, $P\left({ }^{3} \mathrm{He}^{+}\right) \sim P\left({ }^{3} \mathrm{He}\right)=P=p(\beta(e))$ are large enough so that $R P \sim 85 \hbar$ for $R \sim 1.2 \mathrm{~A}$ - the $\mathrm{CH}$ bond length. We can then approximate the motion of the heavy particles on scales larger than, say, $0.5 \mathrm{~A}$ as classical and deterministic. By the late time that this distance is reached, the Born Oppenheimer approximation may become applicable. [25]

The graphene sheet will then behave as an ideal conductor with the electronic distribution mimicking a negative image charge that is situated at $(-Z)$. The resulting attractive potential energy $\mathcal{V}^{\prime}=-e^{2} /(2 Z)$ assumes at $Z=$ $1.1 \mathrm{~A}=2 a_{\text {Bohr }}$ the value of $\sim 0.5 \mathrm{Ry}=6.8 \mathrm{eV}$. This potential energy is much larger, in absolute value, than the initial kinetic energy $\sim 1.85 \mathrm{eV}$ (the free recoil energy $\sim 3.7 \mathrm{eV}$ minus the binding energy of $\mathrm{B} \sim 1.85 \mathrm{eV}$ ). As such, electrostatic interactions will reverse the motion of the outgoing helium ion. We will not follow the full history of the motion of the helium ion in this one-dimensional (solvable) $1 / Z$ potential. We must remark, however, that the probability of eventually having a final $\mathrm{He}$ atom, is greatly enhanced beyond the fraction of helium atoms in the "helium branch" directly generated by large overlaps in the sudden approximation. This enhancement is due to late captures of a graphene electron by the helium ion upon its multiple reentries into (or reflections from) the graphene 
sheet. In successive encounters, the helium ion also keeps losing its kinetic energy making it eventually stop in a bound state within the graphene.

As we emphasized earlier, such energy exchange will not be reflected in the measured $\beta$ electron spectrum. The late formation of helium atoms will, furthermore, not contribute to the "good" part of the $\beta$ spectrum associated with helium atom branch. At long times, the coherence and the entanglement are lost. These would show up, however, in coincidence experiments which will indicate a very high percentage of events with helium atom, rather than a helium ion in the final state. In the above scenario, the helium ion will survive only in the rare instances where the initial energy of the ZPM of the tritium was so large that the helium ion it converted into can escape. Similar multiple encounters with late ${ }^{3} \mathrm{He}$ formation can happen also in the molecular case thereby enhancing the measured fraction of helium atoms and reducing that of helium ions in the ultimate final state.

\section{SUMMARY AND CONCLUSIONS}

This work was inspired by the claim of [3] that quantum uncertainty poses a fundamental limitation for detecting CNB. Specifically, Ref. [3] underscored that zero point motion (ZPM) induced smearing of the energies of the $\beta$ electrons block experiments aiming to discover the CNB made of neutrinos of masses $\leq 0.1 \mathrm{eV}$ by using tritium bound to a graphene or to other surfaces. If true, this presents a notable obstacle to experiments that aim to measure the mass of light neutrinos. Recalculating the effect, we found that it is significantly smaller than the suggestion of [3]. It still remains a challenge for the lighter $\leq 0.1 \mathrm{eV}$ CNB neutrinos. We supported this effect by kinematic arguments and by recalling two instances of high energy scattering in which atomic or nuclear ZPM play important roles. We also noted that the ZPM due to the phonon degree of freedom of the graphene slightly augments the broadening due to the direct ZPM of the tritium.

Much of our effort was directed towards estimating the broadening due to electronic excitations induced in the graphene by the "sudden" disappearance of a unit of negative electric charge due to the escape of the $\beta$ electron. This was done along with the simpler situations of atomic and molecular ${ }^{3} \mathrm{H}$ within a unified approach where all smearing are viewed as being due to excitations (or more general energy changes) of the system hosting the initial radioactive tritium. Our estimates suggest that electronic excitations of the graphene generate a much larger smearing of the measured energy than the above ZPM effects.

Can we, following a similar suggestion in Ref. [3], ameliorate the smearing by using appropriate materials to which the tritium may be attached? Platinum readily adsorbs hydrogen but binds it more weakly than graphene with a potentially reduced smearing due to the ZPM of the tritium. Unfortunately, the surfaces of the thin platinum sheets are not a regular as those in graphene.

In general, it will be difficult to avoid at the same time excessive smearing due to the ZPM of the tritium atom and the smearing due to a dense spectrum of low energy electronic excitations of the host material. Our quest may thus lead us back to free atomic tritium which, as we saw above, is effectively free from smearing. To approach a state of affairs emulating atomic tritium we have to a) spatially separate the tritium atoms so that they will not combine into molecules, and b) the tritium atoms should be only weakly coupled to an embedding "matrix" material used as otherwise the electronic excitations of the latter could generate excessive smearing.

The desire to fulfill both demands then suggests the following setup. Inspired by the dark matter search experiment using DNA strands hanging from gold sheets suggested some time ago [26], we would like to have the tritium atoms weakly attached to the ends of polymer chains hanging from a thin sheet. If neighboring polymer segments attached to the matrix sheet are (evenly) spaced by a distance $d$ exceeding the common length $l$ of the segments then spontaneous formation of $T_{2}$ molecules will be avoided regardless of how much the segments keep thermally dangling. We still may need to verify that the affinity of the tritium to the matrix sheet will be minimal (far smaller than that of in the above discussion of graphene) in order to avoid the sticking of the tritium ends to the surface after accidental looping back of the polymer chain so as to touch this surface. At distances $\geq 50$ A, electronic excitations in the matrix sheet will be largely decoupled from the far-away tritium and a potentially large resulting smearing - similar to that found above for tritium attached directly to a graphene sheet-will be avoided.

Finally, if the bond via which the tritium is attached to the end of the polymer chain is weak and the corresponding binding potential is very shallow the ZPM of the tritium may be significantly reduced and the attendant smearing far less than the $0.24 \mathrm{eV}$ value found above. None of the dangling and backward looping was a concern in the setup suggested in [26]. The very long and heavy DNA chains will not freely dangle. This is because the gravitational energy in such setups exceeds the thermal energy $k_{B} T$ limiting the amplitude of upward thermal fluctuations [27]. An important factor avoiding such upward jumps of the ends of even short DNA segments as in the present case is the stiffness of the double helical DNA. With DNA, we can further harness bioprocesses (such as PCR) for mass production of the segments and possibly also for the attachment of the segments to the matrix sheet [28]. While the suggested setup seems to be rather complicated and difficult to implement, the goal of discovering the CNB neutrinos and the absolute scale of neutrino masses may well be worth it. 


\section{ACKNOWLEDGMENTS}

It is a pleasure to thank Angelo Esposito, Erik Henriksen, Xiaobo Lu, Chris Tully, and Li Yang and for numerous discussions, careful reading of our work, and illuminating questions and comments (including a very detailed helpful note by Xiaobo $\mathrm{Lu}$ and $\mathrm{Li}$ Yang). We are extremely indebted to Chris Tully for a zoom conversation in which he explained the below "Note added" point. We enjoyed further help and encouragement of Robert Shrock. We wish to acknowledge the Aspen Center for Physics, which is supported by NSF Grant No. PHY-1607611.

Note added.-Following earlier work, we have considered in this paper the case where the concentration of the tritium atom adsorbed on the graphene surface is low so that the latter still remains a semimetal. Chris Tully pointed out to us that in the opposite extreme case of saturation (which may well be experimentally feasible), the initially "free" electrons in the graphene may become bound to the extra covalent hydrogen bond so that the graphene sheet ceases to be a semimetal [30]. This impacts the specific estimate of the broadening effect due to electrons in the actual planned experimental setup. The main goal of our paper was, however, to lay out the conditions for avoiding quantum broadening that are not influenced by this. We also believe that the hierarchy of such difficulties, which keep increasing with the complexity of the system (starting from atomic tritium, to molecular tritium, and culminating in the many body highly complex case of the tritium atoms adsorbed of graphene) remains.

Recently, Ref. [3] was expanded and published with a new title [31]. The revised contents of this new work do not impact our analysis and conclusions. In particular, the original estimate of the broadening in [3], which we show [Eq. (6)] to be erroneous (by more than a factor of 3 due to the confluence of two specific errors in [3]), remains unchanged in [31].

The final message of our paper is that a dedicated highly complex many body calculations of the various energy eigenstates and overlaps arising in the sudden approximation should be undertaken. The ingenuity, sophistication, and importance of the Ptolomey experiment do call for a matching theoretical effort dedicated to studying the actual experimental situation.
[1] L. I. Bodine, D. S. Parno, and R. G. H. Robertson, Assessment of molecular effects on neutrino mass measurements from tritium $\beta$ - decay, Phys. Rev. C 91, 035505 (2015).

[2] L. I. Bodine, Molecular effects in tritium beta-decay neutrino-mass measurements, Ph.D. thesis, University of Washington, Seattle, 2015, https://inspirehep.net/files/ 3f5ac132b0ce5d1999616c4bb3ad073d.

[3] Y. Cheipesh, V. Cheianov, and A. Boyarsky, Heisenberg's uncertainty as a limiting factor for neutrino mass detection in $\beta$-decay, https://arxiv.org/pdf/2101.10069v2.pdf (January 2021).

[4] E. Baracchini et al., PTOLEMY: A proposal for thermal relic detection of massive neutrinos and directional detection of MeV dark matter, arXiv:1808.01892.

[5] N. Aghanim et al. (Planck Collaboration), Planck 2018 results. VI. Cosmological parameters, Astron. Astrophys. 641, A6 (2020).

[6] K. Akita, S. Hurwitz, and M. Yamaguchi, Precise capture rates of cosmic neutrinos and their implications on cosmology, Eur. Phys. J. C 81, 344 (2021).

[7] To be precise, this holds true when only a small fraction of the sites are occupied and also in the opposite situation where the surface is completely saturated with tritium.

[8] M. Moaied, J. Moreno, M. Caturla, F. Yndurain, and J. Palacios, A theoretical study of the dynamics of atomic hydrogen on Graphene bilayers, arXiv:1405.3165.

[9] D. Henwood and J.D. Carey, $A b$ initio investigation of molecular hydrogen physisorption on graphene and carbon nanotubes, Phys. Rev. B 75, 245413 (2007).
[10] H. González-Herrero, E. Cortés-del Río, P. Mallet, J.-Y. Veuillen, J. J. Palacios, J. M. Gómez-Rodríguez, I. Brihuega, and F. Ynduráin, Hydrogen physisorption channel on graphene: A highway for atomic $\mathrm{H}$ diffusion, 2D Mater. 6, 021004 (2019).

[11] I. Esteban, C. G. Garcia, M. Maltoni, T. Schwetz, S. Thomas, and Z. Albert, Parameter ranges (2020), www .NuFIT.org, Three-neutrino fit (NuFIT 3.2 ed.).

[12] The momentum transfers in the CNB capture reaction are much lower than the $\sim 100 \mathrm{Mev}$ nuclear scale momenta. This allows treating the ${ }^{3} \mathrm{H}$ and ${ }^{3} \mathrm{He}$ spin half nuclei as pointlike fermions. The $(V-A) X(V-A)$ local four fermion weak interaction can be Fierz rearranged into a $(V-$ A) $X(V-A)$ form in the crossed (s) channel,

$\bar{\psi}^{3} \mathrm{He}^{+}(x) \gamma_{\mu}\left(1-\gamma_{5}\right) \psi_{\nu(e)}(x) \cdot \bar{\psi}^{3} \mathrm{H}(x) \gamma_{\mu}\left(1-\gamma_{5}\right) \psi_{e}(x)$.

This form clearly exhibits the transition from the initial state I of $\nu_{e}$ and ${ }^{3} \mathrm{H}$ into the final state of ${ }^{3} \mathrm{He}^{+}$and $e^{-}$.

[13] X. Cong, Q.-Q. Li, X. Zhang, M.-L. Lin, J.-B. Wu, X.-L. Liu, P. Venezuela, and P.-H. Tan, Probing the acoustic phonon dispersion and sound velocity of Graphene by Raman spectroscopy, Carbon 149, 19 (2019).

[14] M. G. Aartsen, R. Abbasi et al. (The IceCube Collaboration), Detection of a particle shower at the Glashow resonance with IceCube, Nature (London) 591, 220 (2021).

[15] S. L. Glashow, Resonant scattering of antineutrinos, Phys. Rev. 118, 316 (1960). 
[16] A. Loewy, S. Nussinov, and S. L. Glashow, The effect of doppler broadening on the $6.3 \mathrm{PeV} W^{-}$resonance in $\bar{\nu}_{e} e^{-}$ collisions, arXiv:1407.4415.

[17] The reason for the earlier noted "decoherence" is that the phases $\left(E_{n}^{\prime}-E_{0}^{\prime}\right) t / \hbar$ in Eq. (26) become large. An analog of the basic quantum mechanical "collapse" may then sets in. The system then ceases to be a single wave function with a coherent superposition of the different energy states. The system may effectively become a statistical mixture of the different energy states. The spectrometer measurement finally forces the system to be in one of its energy eigenstates.

[18] This condition is motivated by the atomic tritium beta decay where, as we will show below [Eq. (27)], the relevant overlap is 0.64 and the experiment can proceed with minimal degradation.

[19] F. Boehm, P. Vogel, and P. Vogel, Physics of Massive Neutrinos (Cambridge University Press, Cambridge, England, 1992).

[20] G. Feinberg, J. Sucher, and C. K. Au, The dispersion theory of dispersion forces, Phys. Rep. 180, 83 (1989).

[21] A. A. Evett, Ground state of the helium hydride ion, J. Chem. Phys. 24, 150 (1956).

[22] A. M. Schwartz, Excitations of molecules in the $\beta$ decays of constituent atoms, J. Chem. Phys. 23, 400 (1955).

[23] M. Aker et al. (KATRIN Collaboration), Improved Upper Limits on the Neutrino Mass from a Direct Kinematic Method by KATRIN, Phys. Rev. Lett. 123, 221802 (2019).

[24] L. Yang, J. Deslippe, C.-H. Park, M. L. Cohen, and S. G. Louie, Excitonic Effects on the Optical Response of Graphene and Bilayer Graphene, Phys. Rev. Lett. 103, 186802 (2009).

[25] The Born-Oppenheimer approximation is not valid at short times following the sudden escape of the $\beta$ electron. The much faster motion of the atomic electrons relative to the slow nuclei justifies solving for their instantaneous wave function and energy for fixed nuclear positions. However, employing the lowest energy eigenvalue as an effective potential for determining the nuclear motions may be invalid here. Initially, the system is not in the ground state of $\mathcal{H}^{\prime}$. Instead, there is a superposition of many nearby energy eigenstates; the nuclei will not move in a unique
Born-Oppenheimer potential but in a superposition of many such potentials.

[26] A. K. Drukier, Ch. Cantor, M. Chonofsky, G. M. Church, R. L. Fagaly, K. Freese, A. Lopez, T. Sano, C. Savage, and W. P. Wong, New class of biological detectors for WIMPs, Int. J. Mod. Phys. A 29, 1443007 (2014).

[27] Conceivably, the electrically conducting DNA features a dense spectrum of electronic excitations that undermine our suggestion. A possible, admittedly rather difficult to implement, solution to the problem of backwards looping and sticking could be the following. If the length of the flexible polymer segment at the end of which the tritium is located is at $\ell \simeq 50$ Angstrom then to avoiding thermal backward looping, we need to add a mass element $m$ at (or near) the tip such that $m g h \geq k_{B} T$. For $T \sim 1$ Kelvin, a mass of $m \simeq$ $10^{-12}$ gram which is that of a spherical iron grain of radius $R \simeq 3 \times 10^{-5} \mathrm{~cm}$

[28] The possibility of using $\beta$ decaying isotopes other than tritium to study neutrinos has been discussed several times. (For a recent suggestion, see [29].) Isotopes undergoing super-allowed $\beta$ decays like tritium but with lower $\mathrm{Q}$ value would have an enhanced (by $[\Delta / Q]^{3}$ ) fractional population of the near endpoint region of interest of electrons of energies $(Q-\Delta) \leq \epsilon(\beta(e)) \leq Q$. The effect of a fixed neutrino mass would be much larger and $\beta$ spectrometers with weaker electric fields will be required. Also the ZPM of the heavy Isotope and attendant smearing decrease like the inverse of the mass of the isotope. Unfortunately we do not have such isotopes with lower Q values which are still super-allowed. The well known ${ }^{187} \mathrm{Re}$ isotope with $Q=$ $2.5 \mathrm{KeV}$ has a a forbidden transition with too low decay rates.

[29] P. F. Smith, Proposed experiments to detect keV range sterile neutrinos using energy-momentum reconstruction of beta decay or K-capture events, New J. Phys. 21, 053022 (2019).

[30] F. Zhao, Y. Raitses, X. Yang, A. Tan, and C. G. Tully, High hydrogen coverage on graphene via low temperature plasma with applied magnetic field, Carbon 177, 244 (2021).

[31] Y. Cheipesh, V. Cheianov, and A. Boyarsky, Navigating the pitfalls of relic neutrino detection, Phys. Rev. D 104, 116004 (2021). 